\title{
Classifying retrieval strategies as a function of working memory
}

\author{
Jenni L. Schelble • David J. Therriault • \\ M. David Miller
}

Published online: 18 October 2011

(C) Psychonomic Society, Inc. 2011

\begin{abstract}
Strategy selection may help explain performance differences between individuals with high working memory capacity (HWMs) and low working memory capacity (LWMs) (Budd, Whitney, \& Turley, (Memory \& Cognition, 23, 735748 1995); Cokely, Kelley, \& Gilchrist, (Psychonomic Bulletin \& Review, 13, 991-997 2006). We compared the independent and spontaneous strategy use of HWMs and LWMs during a category fluency (retrieval) task that required participants to retrieve animal names. HWMs were more successful at the fluency task under normal conditions, but under increased cognitive load, there were no WM-related performance differences. One strategy (i.e., retrieving animals according to their scientific classification) significantly aided performance, irrespective of cognitive load. Under normal conditions, HWMs were more likely to use the effective strategy; however, under load, WM did not predict strategy use. Use of the classification strategy was more strongly related to retrieval performance than was WM. These results suggest that retrieval strategy use is related to WM capacity, and that employing a successful strategy may make up for WM disadvantages during a demanding retrieval task.
\end{abstract}

Keywords Working memory · Attention · Individual differences $\cdot$ Semantic memory

Working memory (WM) is a psychological construct that describes an individual's ability to keep information in an active state while also doing work upon that information

\footnotetext{
J. L. Schelble $(\square) \cdot$ D. J. Therriault $\cdot$ M. D. Miller

School of Human Development and Organizational Studies

in Education, University of Florida,

1215 Norman Hall, P.O. Box 117049,

Gainesville, FL 32611, USA

e-mail: tojenni@ufl.edu
}

(Baddeley, 1986; Baddeley \& Hitch, 1974). A large body of research has highlighted the importance of WM in the performance of complex tasks such as reading and reasoning (Conway, Cowan, Bunting, Therriault, \& Minkoff, 2002; Daneman \& Carpenter, 1980; Kane et al., 2004). Unsworth and Engle (2007) argued that WM is necessary for two basic cognitive functions: maintenance of information and retrieval of information from secondary memory. Maintenance consists of selective attention (i.e., focus in the presence of taskirrelevant distractors) and/or controlled attention (e.g., providing participants with to-be-recalled information under load or having them engage in a dual task). Retrieval from secondary memory uses controlled search processes to distinguish between task-relevant and task-irrelevant information. Unlike the bulk of attention research, which has explored issues related to maintenance, the present study focuses on retrieval. Specifically, we examined the retrieval strategies of individuals as a function of their WM capacity, exploring the possibility that different strategies may be paired with high or low WM capacity.

Strategy selection (used to retrieve information from secondary memory) may partially explain individual differences in retrieval (Budd, Whitney, \& Turley, 1995; Cokely, Kelley, \& Gilchrist, 2006; McNamara \& Scott, 2001; Rosen \& Engle, 1997). It might be the case that individuals with high WM capacity (HWMs) and individuals with low WM capacity (LWMs) use different strategies during demanding tasks, due to the capacity they have available for employing strategies (Kaakinen \& Hyönä, 2007; Linderholm, Cong, \& Zhao, 2008; Rosen \& Engle, 1997). However, we still know little about the specific strategies that individuals employ spontaneously during retrieval tasks.

Rosen and Engle (1997) showed that retrieval decline (due to increased cognitive load) occurs only in HWM participants. In their study, participants were asked to 
generate a list of animal names without repeating any animal. Under memory load, HWMs had more difficulty producing unrepeated animal names than did LWMs, suggesting that HWMs are unable to suppress previously retrieved responses because they are unable to attend to them. One possible explanation for this result is the automation of retrieval tasks - LWMs' retrieval was more automatic, as evidenced by the lack of load effects on LWMs' performance. Under nonload conditions, the greater WM capacity of HWMs enabled them to "strategically generate their own cues for greater recall” (p. 225). When not under memory load, HWMs retrieved more animal names, as well as more animal clusters (i.e., groups of semantically related words separated by pauses). Although strategy use was suggested as a possible explanation for WM group performance differences during the retrieval task, specific strategies used by participants have not been examined.

Our goals in conducting the present research were to gather information on the types of strategies that participants employ during a retrieval task (i.e., naming animals) and then to determine whether individuals employ these strategies differently as a function of their WM capacity. The task of generating animal names can be approached in many different ways (e.g., going through the alphabet, thinking of a certain environment and naming all of the animals that live there, or using a scientific classification to generate animals, such as types of mammals or types of dogs). Previous research has documented a relationship between WM and performance on the animal-naming task (Rosen \& Engle, 1997). HWM and LWM individuals use different strategies for some tasks (Kaakinen \& Hyönä, 2007; Linderholm et al., 2008), and previous researchers have suggested that strategic recall may be responsible for WM-related differences in retrieval performance (Rosen \& Engle, 1997). Because they posited that retrieval differences could be attributed to differences in strategy use, we think that it is important to begin to document the strategies that individuals use during retrieval.

Another goal in exploring the relationship between WM and strategy use is to begin to explain why HWMs outperform LWMs. We believe that differences in strategy use are best characterized by difference in either kind or degree. This distinction leads to four possible explanations for strategy-related performance differences between HWMs and LWMs on retrieval tasks:

- HWMs use fundamentally different strategies than LWMs do (difference in kind).

- HWMs and LWMs use the same strategies, but HWMs use the strategies more efficiently/productively (difference in degree).

- HWMs and LWMs use the same pool of strategies, but HWMs employ a larger repertoire (more) of the strategies (difference in degree).
- HWMs and LWMs use many of the same strategies, but HWMs employ the strategies more productively. HWMs also have additional strategies at their disposal that are not available to LWMs (difference in kind and degree).

We hypothesized that the fourth option above would most accurately describe the relationship between WM and strategy use. Differences in strategy use as a function of WM have been reported for some tasks (Budd et al., 1995; Cokely et al., 2006; McNamara \& Scott, 2001), but general attentional capacity is a strong predictor of performance on many cognitive tasks, even those for which strategy use (TurleyAmes \& Whitfield, 2003) and previous knowledge (Conway \& Engle, 1996) are controlled. Consequently, WM capacity should positively impact both efficiency (i.e., more efficient strategy use) and the number of strategies employed (i.e., more strategies selected). To our knowledge, no previous data on category fluency task strategies exist, so we had no predictions about which specific strategies HWMs and LWMs would use. However, Rosen and Engle's (1997) cluster analysis suggested that HWMs and LWMs approached the retrieval task differently (i.e., HWMs named both more clusters of animals and more animals overall), providing support for the idea that strategies may be responsible for WM-related performance differences.

Although significant retrieval performance differences emerge between HWMs and LWMs under normal conditions, when cognitive load is introduced, HWMs' performance suffers (Cokely et al., 2006; Rosen \& Engle, 1997). Load appears to influence HWMs' general performance, as well as their strategy selection (Cokely et al., 2006). During a list recall task that required participants to suppress irrelevant cue words, several of the highest-WM participants reported attempting to avoid looking at, or processing, the cue words. When participants' ability to avoid cue words was controlled in the second experiment, HWMs exhibited large interference effects, while LWMs exhibited none. This suggests that HWMs and LWMs approach recall tasks using different strategies, and that cognitive load negatively affected HWMs' strategy effectiveness. Cokely et al. suggested that "individual differences in attentional control are not solely differences in the capacity to control attention, but can stem from better strategic allocation of executive resources" (p.991). In the present experiments, we sought to identify these possible strategies in a retrieval task, and to determine whether they are qualitatively or quantitatively different as a function of WM capacity.

Rosen and Engle (1997) described differences between their WM groups as a function of strategic (employed by HWM participants) or automatic (employed by LWM participants) cue retrieval. In addition to affecting individuals' tendency to rely on certain types of strategies, WM capacity may influence strategy success when HWM and LWM 
individuals employ the same strategies (i.e., if an HWM individual and an LWM individual both employ Strategy A, we would expect the HWM individual to perform better on the task for which Strategy A is used, if WM capacity affects strategy success). According to the adaptive strategy choice model (Siegler \& Shipley, 1995), people consider the effectiveness of a strategy when deciding whether to employ it. Whether someone views a strategy as successful, then, could be related to their perception of the task and their perception of their ability to employ that strategy.

Retrieval does not occur the same way for every task, as is evidenced by differences between facts retrieved during addition and subtraction tasks (Barrouillet, Mignon, \& Thevenot, 2008). Our present study examined specific types of retrieval from long-term memory during a nonmathematical task. In determining what kinds of retrieval strategies participants would select independently, we were especially interested in the breakdown of strategy selection as a function of WM capacity. Using a questionnaire on strategy use, we were able to compare the strategies participants selected according to their overall success on a retrieval task.

\section{The present study}

We sought to extend Rosen and Engle's (1997) original animal-naming task by including a think-aloud protocol in order to gain insight into the range of strategies participants generated independently. We then used the strategies generated by participants to create a comprehensive list of possible strategies for use on a questionnaire. Strategy use, via the questionnaire, was examined in a no-load and a highload experiment. We predicted that HWMs would have additional strategies at their disposal that were not available to LWMs, resulting in HWMs using a greater number of strategies than LWMs. We expected those additional strategies to be more successful than LWMs' strategies (measured by performance on the retrieval task). We also expected HWMs to be more successful than LWMs when both groups used the same strategies (i.e., HWMs would employ the strategies more productively). Under high-load conditions, we predicted that HWMs would experience "choking" more frequently than LWMs, resulting in no significant differences in the number of animals named as a function of WM capacity when participants were under load.

\section{Experiment 1}

The purpose of Experiment 1 was to obtain a comprehensive list of strategies to include on the questionnaire administered during Experiments $2 \mathrm{a}$ and $2 \mathrm{~b}$.
Method

Participants A sample of 24 participants was assigned to the think-aloud experiment. In each of our experiments, students received course credit in exchange for their participation.

Procedure Permission was obtained to audio-record each participant. Participants were informed that they would be recorded for $5 \mathrm{~min}$, and that they should try to continue naming animals for the entire recording session. Participants were also instructed to verbalize any methods they were using to help themselves think of animals.

Results and discussion

Prior to administering the think-aloud task, a priori, the experimenters developed a list of possible strategies (please see Table 1). From the results of the think-aloud, we concluded that our list captured the extent of strategies that could be used. To ensure interrater reliability, both researchers independently assessed the think-aloud transcripts to determine which strategies participants used. Interrater agreement was obtained on $84 \%$ of the think-aloud transcripts (i.e., both researchers agreed on the number and type of strategies in $84 \%$ of the think-aloud transcripts). Any transcripts for which both authors did not report identical strategies were discussed until a consensus was reached. Appendix A contains excerpts of strategy use from the think-aloud transcripts.

Examples of the most frequently used strategies included classification (e.g., naming types of dogs or mammals), environment (e.g., naming sea animals), and personally relevant animals (e.g., naming types of pets). No participants in Experiment 1 generated strategies that were not on our list; in fact, several of the strategies we generated (e.g., listing all animals with a certain physical characteristic, such as "all animals with four legs") were not used by any participants. Fourteen different strategies were reported by participants in Experiment 1. Table 2 contains a list of the strategies generated by participants in Experiment 1, as well as descriptive statistics on the use of each strategy. Due to the small sample size in Experiment 1 and the descriptive nature of this portion of the study, parametric statistical tests were not used to analyze the think-aloud data.

\section{Experiment 2a}

The purpose of Experiment 2a was to determine whether strategy use was related to WM capacity and retrieval performance. The strategies generated by participants in Experiment 1 were used to construct a strategy questionnaire for use in Experiments 2a and 2b. In Experiment 2a, 
Table 1 Think-aloud participants' use of strategies generated by Schelble and Therriault

\begin{tabular}{ll}
\hline Schelble and Therriault's Strategies & $\begin{array}{l}\text { Participants' Use } \\
\text { of Strategies }\end{array}$ \\
\hline
\end{tabular}

Classification/Organization Cues: Scientific

$\begin{array}{ll}\text { Phylum } & \text { No } \\ \text { Class } & \text { Yes } \\ \text { Order } & \text { No } \\ \text { Family } & \text { No } \\ \text { Genus } & \text { No } \\ \text { Species } & \text { Yes }\end{array}$

Classification/Organization Cues: Fictitious Animals Cartoon characters (Roadrunner, Tasmanian Devil) Yes Monsters (basilisk, hippogriff, Bigfoot) No

Fantasy animals (unicorns, dragons) No

Linguistic Cues

Alphabetical

Yes

Rhyming (Dog, frog; cat, rat, bat) No

Attribute Cues: Physical

Diet (plants, insects, fish, meat) No

Color No

Size No

Number of legs No

Body covering (feathers, scales, fur) No

Poisonousness No

Flight capability No

Nocturnal No

Breathing mechanism No

Winged No

Warm/cold blooded No

Sound made No

Attribute Cues: Location

Geographical location (Africa, Florida) Yes

Environment type (jungle, desert, forest) Yes

Location in zoo Yes

Frequency in natural environment Yes

Attribute Cues: Interactions With Humans

Hunted Yes

Endangered No

Extinct Yes

Eaten by humans $\quad$ Yes

Would eat humans/Dangerous to humans Yes

Farm animal Yes

Domestic animal Yes

Animals participant fears $\quad$ Yes

Animals participant likes Yes

Personally relevant animals (has had as pets, Yes

has seen in the wild, etc.)

Other

Random Yes

Association with previous animal Yes we compared participants' self-reported strategy use according to the number of animals they named and their WM score.

Method

Participants A sample of 143 participants was assigned to Experiment 2a. Of these participants, 14 were excluded due to a high number of accuracy errors on the RSPAN task (see Materials below). Five additional participants were excluded because their animal-naming scores fell more than two standard deviations beyond the mean number of animals named.

Materials We administered strategy use questionnaires constructed from the list of strategies generated by participants in Experiment 1. The purpose of the questionnaire was to determine whether the strategies employed varied by WM capacity. The questionnaire consisted of one open-ended page on which participants were instructed to write down anything they did to help them come up with animals, and one page containing a list of strategies (including examples) that participants could mark to indicate that they used a particular strategy.

A modified and automated version of Daneman and Carpenter's (1980) reading span (RSPAN) task was administered to determine the WM capacity of each participant (Unsworth, Heitz, Schrock, \& Engle, 2005). During the RSPAN task, participants assess the sensibility of sentences while simultaneously attempting to remember a list of letters. Participants view a sentence followed by a prompt to indicate whether or not the sentence was sensible. After choosing "true" or "false," a letter appears on the screen. After several sentence/letter combinations, participants view a screen of letters and must recall the correct letters (in order).

Procedure In Experiment 2a, participants were instructed to begin naming animals and were recorded for $5 \mathrm{~min}$. Next, participants completed questionnaires on the types of strategies they used during the animal-naming task (please see Appendix B). Participants were instructed to complete the open-ended question prior to looking at the "check all that apply" items in order to reduce the possibility of participants' open-ended responses being influenced by exposure to the list of strategies on the second page of the questionnaire. After filling out the questionnaire, participants completed the RSPAN task.

Results and discussion

Twelve of the retrieval strategies and WM were regressed on retrieval performance (two strategies examined in 
Table 2 Strategies generated by participants in Experiment $1(N=24)$

Total Participants Who Used Strategy

\begin{tabular}{llll}
\cline { 2 - 3 } Strategy & Example of Strategy & Number & Percent \\
\hline Classification cues & Types of dogs & 11 & 45.8 \\
Make-believe & Cartoon characters & 1 & 4.2 \\
Alphabetical & Animals that begin with "A” & 4 & 16.7 \\
Geographical location & African animals & 14 & 16.7 \\
Environment type & Jungle animals & 6 & 58.3 \\
Location in zoo & Animals in zoo's bat house & 1 & 25.0 \\
Frequency in natural environment & Endangered animals & 4 & 4.2 \\
Affected by humans & Hunted animals & 8 & 16.7 \\
Live with humans & Farm animals & 1 & 33.3 \\
Likes or fears & Favorite animals & 9 & 4.2 \\
Personally relevant animals & Animals participant has had as pets & 4 & 37.5 \\
Association with previous animal & Snake reminds me of bat & 2 & 16.7 \\
Random & Participant stated he/she was naming animals randomly & 2 & 8.3 \\
Other & & 2 & 8.3 \\
\hline
\end{tabular}

Experiment 1 were excluded from further analyses because they were used by less than $5 \%$ of the participants). Each strategy was dummy-coded in the regression model. All variables were entered into the model in a single step. Table 3 contains the correlation matrix of all variables included in the regression analysis. Table 4 contains the complete regression analysis results of Experiment $2 \mathrm{a}$.

In accordance with the recommendations of Conway et al. (2005), we used partial-credit (total number of items recalled) scoring of performance on the WM span task. RSPAN scores were calculated by totaling the number of letters recalled in the correct order on each trial (i.e., participants received credit for any letters recalled in the correct order; it was not necessary for the entire sequence to be correct). Participants' RSPAN scores ranged from 19 to 74. WM was a significant predictor of retrieval performance (measured by the number of animals named), $b=$ $.190, t(111)=2.15, p<.05$. Higher WM capacity was associated with naming a greater number of animals. The mean number of animals named by participants in Experiment 2a was $49.19(S D=14.18)$.

Further analyses of the strategies revealed that 2 of the 12 strategies employed by participants predicted retrieval performance: the classification strategy (i.e., naming animals according to their genus or species), $b=$ $.339, t(111)=3.96, p<.001$, and the environment strategy (i.e., naming animals according to the type of natural environment they live in), $b=.186, t(111)=2.11, p<.05$. The classification strategy was a stronger predictor of performance than was WM.
In order to explore the relationship between strategy use and WM, we examined correlations between WM and reported strategy use. A significant positive correlation was found between a single strategy and $\mathrm{WM}$, the classification strategy, $r(122)=.22, p<.05$. No other strategies correlated significantly with WM. HWMs outperformed LWMs during the retrieval task but reported using many of the same strategies as LWMs. The classification strategy, however, represents an interesting relationship between WM and strategy use: This strategy appears both to help retrieval performance and to be used more often by individuals with higher WM capacity. Mediation analysis indicated that use of the classification strategy had a mediating effect on the relationship between WM and retrieval performance. Specifically, entering the classification strategy into the regression model, then adding WM as a second predictor reduced the effect of $\mathrm{WM}$ on retrieval performance from $5 \%$ to $2 \%$, $R^{2}=.021, F(1,121)=6.44, p<.05$. This indicates that when participants used classification, it had a stronger effect on their retrieval performance than did WM capacity.

We did not find a significant difference in the number of strategies reported as a function of WM capacity, $b<.001, t(111)=1.83, p>.05$. Thus, our hypothesis that HWMs would use a greater number of strategies than LWMs was not supported. The mean number of strategies reported by participants in Experiment $2 \mathrm{a}$ was $3.64(S D=1.27)$. Table 5 contains the prevalence of strategy use for all participants in Experiment $2 \mathrm{a}$. 


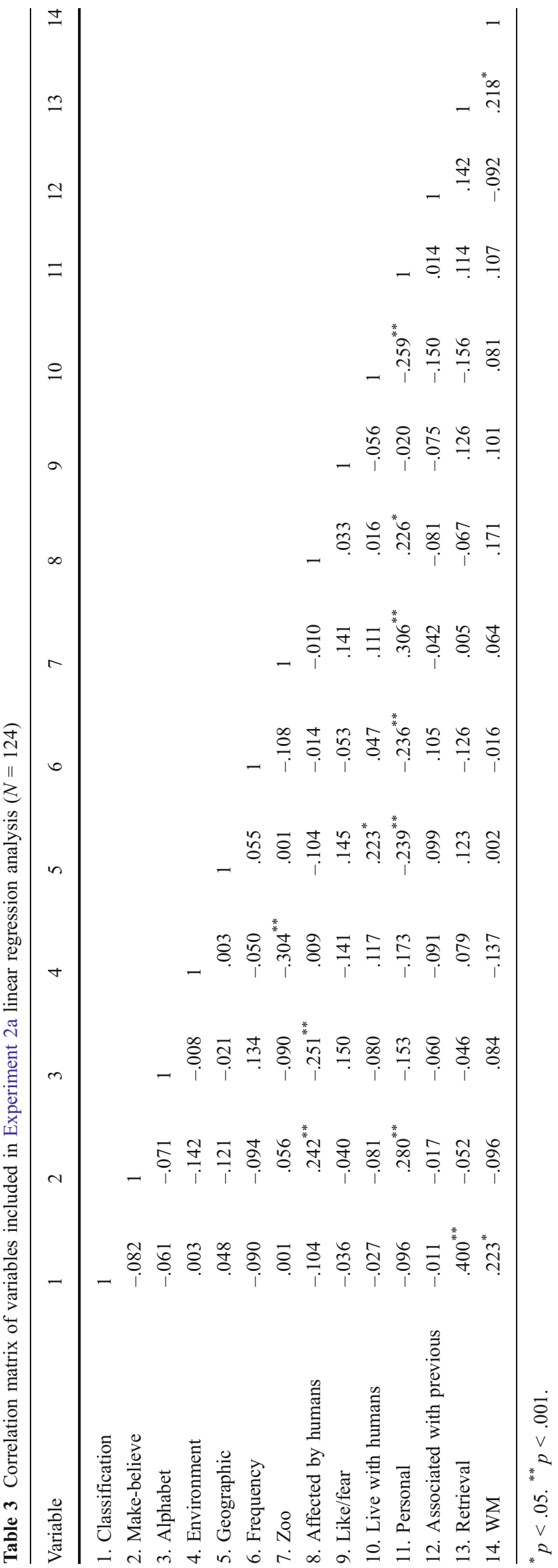

\section{Experiment 2b}

We conducted Experiment $2 \mathrm{~b}$ to further explore Rosen and Engle's (1997) findings that cognitive load would eliminate HWMs' advantage over LWMs during a retrieval task. The purpose of this experiment was to examine the role of attentional capacity in retrieval (i.e., to demonstrate that attentional capacity is directly attributable to performance differences in the task). Additionally, we sought to determine whether cognitive load would affect participants' use of strategies during the retrieval task.

Method

Participants A sample of 99 participants was assigned to Experiment 2b. Five of these participants were excluded due to a high number of accuracy errors on the RSPAN task. Four additional participants were excluded because their retrieval performance scores fell more than two standard deviations beyond the mean. Another 2 participants were excluded due to technical errors.

Materials We used the RSPAN task and strategy questionnaire from Experiment 2a. Additionally, participants completed a digit-tracking task. The digit-tracking task, similar to the task used by Rosen and Engle (1997), required participants to view digits on a computer. The digits 0-9 appeared one at a time in the four corners of a computer screen. The presentation rate for the digits was one per second, and the digit sequence occurred clockwise around the perimeter of the screen. Participants were told to press the space bar on the computer keyboard whenever they saw a third odd digit in a row appear in the sequence. The program registered responses and provided feedback depending on the responses. When participants saw three odd digits in a row, they were instructed to press the space bar. Incorrect presses or missed presses resulted in a screen that read "Please try harder."

Procedure In Experiment 2b, participants practiced the digit-tracking task before naming animals. Participants practiced the task for $1 \mathrm{~min}$. After ensuring that the participant understood the task, the researcher instructed the participant to name animals out loud while completing the digit-tracking task. Participants were audio-recorded during the task, and permission to record each participant was obtained. The digit-tracking task ran for $5 \mathrm{~min}$. Participants were instructed to continue naming animals for the entire duration of the task. They then completed strategy questionnaires identical to those completed by the participants in Experiment 2a. Finally, participants completed the RSPAN task. 
Table 4 Linear regression of variables related to retrieval performance in Experiment 2a $(N=124)$

\begin{tabular}{|c|c|c|c|}
\hline Variable & $B$ & $S E B$ & $\beta$ \\
\hline \multicolumn{4}{|c|}{ Retrieval strategies: $(1=$ participant reported using strategy, $0=$ participant did not report using strategy $)$} \\
\hline Classification & .097 & .024 & $.339^{* *}$ \\
\hline Make-believe & .002 & .025 & .006 \\
\hline Alphabet & -.007 & .025 & -.023 \\
\hline Environment & .053 & .025 & $.186^{*}$ \\
\hline Geographic location & .037 & .025 & .129 \\
\hline Frequency & -.030 & .045 & -.058 \\
\hline Zoo location & .004 & .024 & .014 \\
\hline Affected by humans & -.026 & .033 & -.070 \\
\hline Liked or feared animals & .081 & .056 & .124 \\
\hline Live with humans & -.031 & .023 & -.121 \\
\hline Personally relevant & .037 & .026 & .143 \\
\hline Associated with previous animal & .057 & .033 & .146 \\
\hline \multicolumn{4}{|l|}{ Working memory measure: } \\
\hline RSPAN score & .003 & .001 & $.190^{*}$ \\
\hline
\end{tabular}

${ }^{*} p<.05{ }^{* *} p<.001$.

Results and discussion

The mean number of animals named by participants in Experiment $2 \mathrm{~b}$ was $35.2(S D=12.12)$. An independentsamples $t$ test revealed that participants in Experiment $2 \mathrm{~b}$ named significantly fewer animals than did participants in Experiment 2a, $t(210)=-6.09, p<.001$. The mean number of strategies reported by participants in Experiment $2 \mathrm{~b}$ was $2.28(S D=1.14)$, which was significantly fewer than the strategies reported by participants in Experiment 2a, $t(210)=$ $-4.47, p<.001$.

Participants' RSPAN scores ranged from 26 to 75 . Consistent with Rosen and Engle's (1997) findings, there were no significant differences in the numbers of animals named as a function of WM in the load condition, $b=.014, t(74)=0.094$, $p>.05$. As expected, load removed HWMs' advantage during the retrieval task, causing them to underperform. HWM participants in Experiment 2a named significantly more animals than did HWM participants in Experiment 2b, $t(55)=$ $-5.12, p<.001$. This evidence lends support to the theory that the additional capacity HWMs rely on for their superior performance is not available when increased cognitive load takes up HWMs' formerly excess resources.

Analysis of participants' strategy use in Experiment $2 b$ revealed that no strategies correlated significantly with WM. Please see Table 6 for the complete correlation matrix
Table 5 Prevalence of strategies in Experiment 2a $(N=124)$ and $2 \mathrm{~b}(N=88)$

\begin{tabular}{lllllll}
\hline & \multicolumn{2}{l}{ Experiment $2 \mathrm{a}$} & \multicolumn{2}{l}{ Experiment $2 \mathrm{~b}$} & \multicolumn{2}{l}{ Total } \\
\cline { 2 - 7 } Strategy & No. & $\%$ & No. & $\%$ & No. & $\%$ \\
\hline Classification & 35 & 28.0 & 31 & 35.6 & 66 & 31.1 \\
Make-believe & 36 & 28.8 & 13 & 14.9 & 49 & 23.1 \\
Alphabet & 34 & 27.2 & 9 & 10.3 & 43 & 20.3 \\
Environment & 89 & 71.2 & 46 & 52.9 & 135 & 63.7 \\
Geographic location & 35 & 28.0 & 14 & 16.1 & 49 & 23.1 \\
Frequency of animal & 8 & 6.4 & 6 & 6.9 & 14 & 6.6 \\
Location of animal in zoo & 57 & 45.6 & 35 & 40.2 & 92 & 43.4 \\
Animal affected by humans & 18 & 14.4 & 6 & 6.9 & 24 & 11.3 \\
Animals participant likes/fears & 5 & 4.0 & 4 & 4.6 & 9 & 4.2 \\
Animals that live with humans & 67 & 53.6 & 41 & 47.1 & 108 & 50.9 \\
Personally relevant animals & 56 & 44.8 & 27 & 31.0 & 83 & 39.2 \\
Associated with previous animal & 15 & 12.0 & 19 & 21.8 & 34 & 16.0 \\
\hline
\end{tabular}


Table 6 Correlation matrix of variables included in Experiment $2 \mathrm{~b}$ linear regression analysis $(N=88)$

\begin{tabular}{|c|c|c|c|c|c|c|c|c|c|c|c|c|c|c|}
\hline Variable & 1 & 2 & 3 & 4 & 5 & 6 & 7 & 8 & 9 & 10 & 11 & 12 & 13 & 14 \\
\hline $\begin{array}{l}1 . \\
\text { Classification }\end{array}$ & 1 & & & & & & & & & & & & & \\
\hline $\begin{array}{l}\text { 2. Make- } \\
\text { believe }\end{array}$ & -.110 & 1 & & & & & & & & & & & & \\
\hline 3. Alphabet & .063 & -.037 & 1 & & & & & & & & & & & \\
\hline 4. Environment & -.115 & $-.250^{*}$ & .018 & 1 & & & & & & & & & & \\
\hline 5. Geographic & .001 & .080 & -.046 & -.088 & 1 & & & & & & & & & \\
\hline 6. Frequency & .082 & .013 & .056 & -.016 & -.119 & 1 & & & & & & & & \\
\hline 7. Zoo location & -.023 & -.015 & .029 & $-.212^{*}$ & -.040 & -.131 & 1 & & & & & & & \\
\hline $\begin{array}{l}\text { 8. Affected by } \\
\text { humans }\end{array}$ & -.013 & -.114 & -.092 & .075 & .004 & .105 & $.239^{*}$ & 1 & & & & & & \\
\hline 9. Like/fear & .066 & -.092 & -.075 & -.013 & -.096 & -.060 & .044 & -.060 & 1 & & & & & \\
\hline $\begin{array}{l}\text { 10. Live with } \\
\text { humans }\end{array}$ & -.077 & -.073 & -.018 & .153 & .025 & -.166 & .165 & .107 & .123 & 1 & & & & \\
\hline 11. Personal & -.084 & -.002 & -.146 & $-.263^{*}$ & .044 & -.085 & .108 & .112 & .090 & $-.235^{*}$ & 1 & & & \\
\hline $\begin{array}{l}\text { 12. Associated } \\
\text { with previous }\end{array}$ & .013 & -.065 & -.088 & -.170 & -.080 & .186 & -.150 & -.034 & .150 & -.165 & .006 & 1 & & \\
\hline 13. Retrieval & $.279^{* *}$ & -.038 & .118 & .091 & -.061 & $.234^{*}$ & $-.263^{*}$ & -.021 & -.004 & .089 & -.126 & $.259^{*}$ & 1 & \\
\hline 14. WM & .116 & .182 & .028 & -.098 & -.071 & .036 & .063 & -.035 & -.146 & -.167 & -.007 & .126 & .006 & 1 \\
\hline
\end{tabular}

${ }^{*} p<.05{ }^{* *} p<.001$.

for Experiment 2b. Please see Table 7 for complete regression analysis results of this experiment.

As in Experiment 2a, use of the classification strategy predicted retrieval performance, $b=.710, t(74)=5.24, p<.001$. This suggests that, even under increased cognitive load, the classification strategy was useful for retrieving animals from long-term memory. Under load, however, HWM individuals were no more likely to use this strategy than were LWM individuals, perhaps because they could not access their normally superior attentional resources. Naming animals that live with humans also predicted retrieval performance in Experiment $2 \mathrm{~b}$ (but not in Exp.2a), $b=.322, t(74)=2.08, p<.05$.

We also explored whether the classification strategy became less frequent under load for the highest-WM participants (i.e., those with WM scores in the 75th percentile) using the chisquare test with the Yates correction for continuity. The chisquare test indicated that attempts to use the classification

Table 7 Linear regression of variables related to retrieval performance in Experiment $2 \mathrm{~b}(N=88)$

\begin{tabular}{|c|c|c|c|}
\hline Variable & $B$ & $S E B$ & $\beta$ \\
\hline \multicolumn{4}{|c|}{ Retrieval strategies: $(1=$ participant reported using strategy, $0=$ participant did not report using strategy $)$} \\
\hline Classification & 19.13 & 3.65 & $.710^{* *}$ \\
\hline Make-believe & 2.76 & 4.89 & .077 \\
\hline Alphabet & 6.61 & 5.14 & .171 \\
\hline Environment & 5.94 & 3.59 & .237 \\
\hline Geographic location & 4.87 & 3.80 & .166 \\
\hline Frequency & 3.78 & 9.12 & .064 \\
\hline Zoo location & -3.61 & 3.47 & -.143 \\
\hline Affected by humans & 12.46 & 6.71 & .256 \\
\hline Liked or feared animals & 6.47 & 8.38 & .110 \\
\hline Live with humans & 7.99 & 3.84 & $.322^{*}$ \\
\hline Personally relevant & -2.22 & 4.14 & -.081 \\
\hline Associated with previous animal & -7.17 & 6.62 & -.186 \\
\hline \multicolumn{4}{|l|}{ Working memory measure: } \\
\hline RSPAN score & 1.76 & 18.61 & .014 \\
\hline
\end{tabular}

${ }^{*} p<.05 .{ }^{* *} p<.001$. 
strategy did not differ as a function of cognitive load for individuals in this group, $\chi^{2}(1, N=57)=0.766, p>.05$. Table 5 contains the prevalence of strategy use for all participants in Experiment 2b.

\section{Joint analysis of Experiments 2a and 2b}

We conducted a joint analysis of Experiments $2 \mathrm{a}$ and $2 \mathrm{~b}$ in order to examine the effect of the interaction of WM and cognitive load on retrieval performance.

\section{Method}

Participants Outlier removal procedures conducted with the original data sets of Experiments $2 \mathrm{a}$ and $2 \mathrm{~b}$ combined $(n=$ 221) resulted in the removal of 15 participants, resulting in 206 participants for the combined analysis.

Procedure Retrieval performance data, strategy questionnaire responses, and WM scores from Experiments $2 \mathrm{a}$ and $2 \mathrm{~b}$ were used to complete the joint analysis.

\section{Results and discussion}

A linear regression analysis of the combined $2 a$ and $2 b$ samples was used to examine the effect of the interaction of WM and cognitive load on retrieval performance. The 12 retrieval strategies examined in Experiments $2 \mathrm{a}$ and $2 \mathrm{~b}$, $\mathrm{WM}$, experiment ( $2 \mathrm{a}$ or $2 \mathrm{~b}$ ), and the interaction of WM and experiment were regressed on retrieval performance. Each strategy was dummy-coded in the regression model. The experiment variable was also dummy-coded. All variables were entered into the model in a single step. Consistent with Rosen and Engle's (1997) findings, load interacted with WM capacity to predict retrieval performance, $b=$ $.388, t(190)=2.152, p<.05$. As load increased, the ability of WM to predict retrieval performance decreased. As expected, load removed HWMs' advantage during the retrieval task, causing them to underperform. This evidence lends support to the theory that the additional capacity HWMs rely on for their superior performance is not available when increased cognitive load takes up HWMs' formerly excess resources (Kane \& Engle, 2000).

\section{General discussion}

Strategy use is related to performance differences between HWM and LWM individuals on a variety of tasks (Cokely et al., 2006; Linderholm et al., 2008; McNamara \& Scott, 2001). For some tasks, the most effective strategies cannot be used efficiently by members of both WM groups (Kaakinen \& Hyönä, 2007). Under cognitive load, HWMs' performance suffers, while LWMs' does not (Cokely et al., 2006; Rosen \& Engle, 1997). Differences in strategy use have been proposed as one explanation for these discrepancies. Our results indicate that, during a category fluency task, two strategies predicted retrieval performance under normal conditions. One of those strategies (the classification strategy) was more likely to be used by individuals with high WM capacity. In fact, use of the classification strategy significantly mediated the effect of WM on retrieval performance. Under cognitive load, however, the classification strategy continued to help retrieval performance, but HWMs were no more likely to use this strategy than were LWMs. The classification strategy was a stronger predictor of performance than was any other variable, including WM, when participants were under load. An additional strategy, naming animals that live with humans, appears to help participants under load, but it is not as helpful when participants are not under load. This suggests that individuals may be aware of their limited capacity under load, and may choose different strategies as a result.

Because classification is the only strategy that consistently predicts retrieval performance and HWMs demonstrate retrieval performance superior to that of LWMs, it is not surprising that this strategy is more likely to be used by HWMs. This finding provides support for our hypothesis that HWMs and LWMs would demonstrate strategy differences related to retrieval performance. The WM-related differences in use of the classification strategy indicate that HWMs and LWMs approach retrieval tasks in different ways, and that HWMs' approaches are advantageous. The mediating effect of classification on the relationship between WM and retrieval indicates that use of an effective strategy accounts for more of HWMs' success during this task than does WM capacity alone.

Our study also replicated Rosen and Engle's (1997) finding that HWM individuals "choke" under increased cognitive load. We demonstrated that, under normal conditions, HWMs outperformed LWMs, but when cognitive load was increased, HWMs did no better on the retrieval task than LWMs. HWMs who were not under load named significantly more animals than did HWM participants who completed the retrieval task under load.

Productivity of specific retrieval strategies

Even when WM was a significant predictor of retrieval performance (i.e., in the no-load experiment), the most effective strategy was a stronger predictor of retrieval performance 
than was WM. It is important to note that the majority of strategies participants chose did not result in performance differences, either as a function of WM or in terms of retrieval performance in general. The classification strategy was the exception to both of these findings, because it contributed to retrieval performance and correlated with WM capacity. The considerable effectiveness of the classification strategy when participants were under load demonstrates that using an effective strategy during retrieval can help to compensate for the disadvantages of additional load. The classification strategy is similar to the hierarchical organization of semantic memory described in the original Collins and Quillian (1972) model of semantic memory (e.g., accessing the "dog" category could lead to activation of "types of dogs," a lower-order category below the concept of "dog," or to "cats," which are also contained in the higher-order category of "animals"). The model posits that memory is organized according to networks of ordered categories accessed by spreading activation (i.e., accessing one concept in a network leads to accessing other concepts that are connected to it). Attending to classification, then, may be the most effective strategy for naming animals because it makes use of the way information is organized in the network. Although hierarchical organization is not a component of many current memory models, the concept of spreading activation persists (Lund \& Burgess, 1996; Roelofs, 1992). Additionally, spreading-activation models represent core cognitive processes that do not differ greatly between individuals; our results suggest that WMdemanding tasks tap similarly fundamental processes.

Strategy selection as a function of working memory (differences in kind)

WM capacity is indicative of the types of strategies chosen by individuals for some tasks (Beilock \& DeCaro, 2007) and contributes to the explanation of individuals' strategy choices for our retrieval task. When participants were not under load, HWMs selected the most effective retrieval strategy (classification) more often than LWMs did. However, when participants were under load, WM did not predict effective strategy use. These differences highlight the role of attentional capacity in access to and execution of retrieval strategies.

The absence of a relationship between WM and effective strategy use in the load condition, combined with the significant relationship between strategy use and retrieval performance under load, suggests that LWMs are capable of using effective retrieval strategies. This is an important consideration during strategy instruction (e.g., LWMs and HWMs may be able to use the same methods to retrieve information from secondary memory when studying, but LWMs may require additional practice or study time in order to achieve the same results as HWMs; Linderholm et al., 2008; Linderholm \& Zhao, 2008). LWMs were just as likely as HWMs to use the classification strategy under load. Using the classification strategy appears to compensate for low WM capacity, as classification was more strongly related to retrieval performance than was WM in both experiments. If the classification strategy is useful for other retrieval tasks, training LWMs to use this strategy might improve their performance, particularly when they are under high cognitive load.

General capacity and retrieval performance (differences in degree)

HWMs performed significantly better than LWMs during the retrieval task, in part due to their effective use of the classification strategy. HWMs' superior performance under normal conditions (but not under load) also emphasizes the role of attentional capacity in tasks that require access to secondary memory. Even though the majority of retrieval strategies were used by many participants at each end of the WM range, HWMs named more animals when they were not under load, leading to the conclusion that, when HWMs and LWMs were using the same strategies, HWMs were able to execute strategies more efficiently than LWMs. This finding is consistent with research on encoding strategies (Kaakinen \& Hyönä, 2007) and text-processing tasks (Budd et al., 1995). Although the classification strategy was partially responsible for HWMs' superior performance, it does not explain all of the shared variance between WM and retrieval performance (i.e., mediation analysis indicated that WM still accounted for some of the variance in retrieval performance, even after classification was entered as a predictor of retrieval). However, when HWM participants used the classification strategy, it accounted for more of the variance in their retrieval performance than did WM capacity.

Number of strategies selected as a function of WM capacity

The number of strategies utilized by participants did not vary as a function of WM capacity in either experiment. Thus, one element of our hypothesis (i.e., that HWM participants would utilize a greater number of strategies) was not supported. This contributes to our understanding of the nature of individual differences in WM, which clarifies how HWMs benefit from their additional attentional capacity during retrieval tasks: HWMs do not seem to use 
their additional capacity to access more strategies for the same task; rather, they use the same number of strategies as LWMs, but employ them more successfully (as evidenced by naming more animals than LWMs over a 5 -min period using the same number of strategies). This represents a difference in degree (of strategy effectiveness), as opposed to a difference in kind (of strategy used).

\section{Limitations}

This present study contributes to our understanding of the relationship between WM-related performance differences and strategy use; however, several methodological limitations should be taken into account when considering our results. Because participants self-reported their strategy use after the animal-naming task was completed, we were unable to causally link each strategy with the number of animals named as a result of its use. The limited variability in retrieval performance as a function of WM capacity may be due to the homogeneity of our sample (i.e., college students). Future research should explore retrieval performance in a more diverse sample in order to allow for more widely generalizable conclusions about the relationship between WM capacity and retrieval strategy use. Finally, Rosen and Engle (1997) found retrieval performance differences between HWMs and LWMs during the first minute of the category fluency task. Differences did not emerge in our sample until the fifth minute. We attribute to this to our inclusion of the entire range of participants (i.e., the entire range of WM scores), as opposed to preselecting high- and low-WM participants in an extreme-groups design, as Rosen and Engle did. Extremegroups designs are expected to produce larger effect sizes than designs that examine the entire range of data (Preacher, Rucker, MacCallum, \& Nicewander, 2005).

\section{Conclusion}

Strategy use has a significant effect on retrieval performance across the WM spectrum, despite increased cognitive load. In addition to providing support for the general-capacity view of WM (Engle, Cantor, \& Carullo, 1992), by demonstrating quantitative differences in retrieval task performance between HWMs and LWMs (differences in degree), we have shown that qualitative differences (differences in kind) also exist, in the form of strategy use. We have also attempted to develop a methodology for examining strategy use. Our study represents an initial attempt to examine individuals' specific retrieval processes within the context of WM. Many previous researchers have proposed a relationship between strategy use and WM capacity (Budd et al., 1995; Cokely et al., 2006; Conway et al., 2002; McNamara \& Scott, 2001;
Rosen \& Engle, 1997); we have provided data on the exact strategies utilized by LWMs and HWMs for a retrieval task. Surveying participants on their retrieval strategy use revealed performance-affecting differences in the ways LWMs and HWMs approached retrieval tasks, as well as unexpected similarities in their use of some strategies. Future researchers may find these methods useful for looking at retrieval strategies or at other types of strategy use.

The absence of performance differences under cognitive load further highlights the role of WM in retrieval from secondary memory. Without access to their additional attentional capacity, HWMs are unable to outperform LWMs. By providing evidence of the specific differences between LWMs and HWMs in their approach to retrieval tasks, we hope to contribute to the body of knowledge on individual differences (and similarities) in cognitive control mechanisms. Our exploration of specific strategies, in relation to both WM and retrieval performance, points to the classification strategy as producing superior results for a category fluency retrieval task. Using the classification strategy helped retrieval performance more than did any other strategy, and more than WM capacity. Thus, participants who used the classification strategy performed much better on the retrieval task, regardless of their WM capacity. Future research should explore strategy use during other retrieval tasks in order to determine whether our findings regarding the usefulness of the classification strategy, and differences in LWMs' and HWMs' strategy use overall, transfer to other types of retrieval.

\section{Author note}

We thank Andrew R. A. Conway for his helpful comments on an earlier version of this article.

\section{Appendix A}

Excerpts of think-aloud transcripts from Experiment 1

\begin{tabular}{ll}
\hline Excerpt & Coded As \\
\hline "I'm thinking of doing it alphabetically and & Alphabet strategy \\
thinking of how many animals I can think of & \\
per letter so ant aardvark anteater ..." & \\
"In saltwater there's red snapper, yellow tail & Environment \\
snapper, red grouper ..." & strategy \\
"A rat, a mouse, alligator, those are all animals & Personally \\
that I see in my neighborhood ..." & relevant strategy \\
"Now I'll do reptiles: snakes, alligators, & Classification \\
crocodiles. Frogs are amphibians: salamanders, & strategy \\
lizards. Now, let's see, I'll do birds: toucans, & \\
flamingos, parakeets, parrots ..." & \\
\hline
\end{tabular}




\section{Appendix B}

Questionnaire administered to participants in Experiments $2 \mathrm{a}$ and $2 \mathrm{~b}$

\section{Please explain anything you did to help you think of animals:}

(An entire lined page was provided. The questions below were not revealed until the participant had completed the lined side of the questionnaire).

Which of the following things did you think about to help you think of animals?

Please check all that apply:

$\square$ Types or classifications of animals (for example, thinking about all the types of dogs or types of mammals)

$\square$ Types of make-believe animals (for example, thinking about all the animals you've seen in cartoons)

$\square$ Going through the alphabet (for example, thinking of all animals that start with " $A$ ", then all that start with "B"...)

$\square$ Rhyming (for example, thinking of dog, then thinking of frog because it rhymes with dog) $\square$ Physical attributes of animals (for example, all thinking of all the animals with wings or all animals with 4 legs)

$\square$ Types of environments animals live in (for example, thinking of all the desert animals or all the jungle animals)

$\square$ Geographic location of animals (for example, thinking of all the animals that live in Africa or Florida)

$\square$ Frequency of animals (for example, thinking of the most common animals or the rarest animals)

$\square$ Location of animals in a zoo (for example, thinking of all animals the at Sea World or in the monkey house at the zoo)

$\square$ Animals affected by humans (for example, thinking of all the animals that humans eat or that are endangered)

$\square$ Animals you like, do not like or fear (for example, thinking of a snake you fear, then thinking of a rat because you also fear rats)

$\square$ Animals that live with humans (for example, thinking of all domesticated animals or all the animals on a farm)

$\square$ Animals personally relevant to you (for example, animals you have had as pets, animals you have seen recently)

$\square$ Using free association or random generation (such as saying whatever animals popped into your head)

$\square$ Association with previous animal (for example, "cat reminds me of dog, which reminds me of wolf')

$\square$ Other (Please explain if not listed on other side)

\section{References}

Baddeley, A. (1986). Working memory. Oxford: Oxford University Press, Clarendon Press.

Baddeley, A. D., \& Hitch, G. (1974). Working memory. In G. H. Bower (Ed.), The psychology of learning and motivation: Advances in research and theory (Vol. 8, pp. 47-89). New York: Academic Press.

Barrouillet, P., Mignon, M., \& Thevenot, C. (2008). Strategies in subtraction problem solving in children. Journal of Experimental Child Psychology, 99, 233-251. doi:10.1016/j.jecp.2007.12.001
Beilock, S. L., \& DeCaro, M. S. (2007). From poor performance to success under stress: Working memory, strategy selection, and mathematical problem solving under pressure. Journal of Experimental Psychology, 33, 983-998. doi:10.1037/0278-7393.33.6.983

Budd, D., Whitney, P., \& Turley, K. J. (1995). Individual differences in working memory strategies for reading expository text. Memory \& Cognition, 23, 735-748. doi:10.3758/BF03200926

Cokely, E. T., Kelley, C. M., \& Gilchrist, A. L. (2006). Sources of individual differences in working memory: Contributions of strategy to capacity. Psychonomic Bulletin \& Review, 13, 991997. doi:10.3758/BF03213914 
Collins, A. M., \& Quillian, M. R. (1972). How to make a language user. In E. Tulving \& W. Donaldson (Eds.), Organization of memory (pp. 309-351). New York: Academic Press.

Conway, A. R. A., Cowan, N., Bunting, M. F., Therriault, D. J., \& Minkoff, S. R. B. (2002). A latent variable analysis of working memory capacity, short-term memory capacity, processing speed, and general fluid intelligence. Intelligence, 30, 163-183. doi:10.1016/S0160-2896(01)00096-4

Conway, A. R. A., \& Engle, R. W. (1996). Individual differences in working memory capacity: More evidence for a general capacity theory. Memory, 4, 577-590. doi:10.1080/741940997

Conway, A. R. A., Kane, M. J., Bunting, M. F., Hambrick, D. Z., Wilhelm, O., \& Engle, R. W. (2005). Working memory span tasks: A methodological review and user's guide. Psychonomic Bulletin \& Review, 12, 769-786. doi:10.3758/BF03196772

Daneman, M., \& Carpenter, P. A. (1980). Individual differences in working memory and reading. Journal of Verbal Learning and Verbal Behavior, 19, 450-466. doi:10.1016/S0022-5371(80)90312-6

Engle, R. W., Cantor, J., \& Carullo, J. J. (1992). Individual differences in working memory and comprehension: A test of four hypotheses. Journal of Experimental Psychology. Learning, Memory, and Cognition, 18, 972-992. doi:10.1037/0278-7393.18.5.972

Kaakinen, J. K., \& Hyönä, J. (2007). Strategy use in the reading span test: An analysis of eye movements and reported encoding strategies. Memory, 15, 634-646. doi:10.1080/09658210701457096

Kane, M. J., \& Engle, R. W. (2000). Working-memory capacity, proactive interference, and divided attention: Limits on long-term memory retrieval. Journal of Experimental Psychology. Learning, Memory, and Cognition, 26, 336-358. doi:10.1037/0278-7393.26.2.336

Kane, M. J., Hambrick, D. Z., Tuholski, S. W., Wilhelm, O., Payne, T. W., \& Engle, R. W. (2004). The generality of working memory capacity: A latent-variable approach to verbal and visuospatial memory span and reasoning. Journal of Experimental Psychology. General, 133, 189-217. doi:10.1037/0096-3445.133.2.189

Linderholm, T., Cong, X., \& Zhao, Q. (2008). Differences in low and high working-memory capacity readers' cognitive and metacog- nitive processing patterns as a function of reading for different purposes. Reading Psychology, 29, 61-85.

Linderholm, T., \& Zhao, Q. (2008). The impact of strategy instruction and timing of estimates on low and high working-memory capacity readers' absolute monitoring accuracy. Learning and Individual Differences, 18, 135-143.

Lund, K., \& Burgess, C. (1996). Producing high-dimensional semantic spaces from lexical co-occurrence. Behavior Research Methods, Instruments, \& Computers, 28, 203-208. doi:10.3758/ BF03204766

McNamara, D. S., \& Scott, J. L. (2001). Working memory capacity and strategy use. Memory \& Cognition, 29, 10-17.

Preacher, K. J., Rucker, D. D., MacCallum, R. C., \& Nicewander, W. A. (2005). Use of the extreme groups approach: A critical reexamination and new recommendations. Psychological Methods, 10, 178-192. doi:10.1037/1082-989X.10.2.178

Roelofs, A. (1992). A spreading-activation theory of lemma retrieval in speaking. Cognition, 42, 107-142. doi:10.1016/0010-0277(92) 90041-F

Rosen, V. M., \& Engle, R. W. (1997). The role of working memory capacity in retrieval. Journal of Experimental Psychology. General, 126, 211-227. doi:10.1037/0096-3445.126.3.211

Siegler, R. S., \& Shipley, C. (1995). Variation, selection, and cognitive change. Developing cognitive competence: New approaches to process modeling (pp. 31-76). Hillsdale: Erlbaum.

Turley-Ames, K. J., \& Whitfield, M. M. (2003). Strategy training and working memory task performance. Journal of Memory and Language, 49, 446-468. doi:10.1016/S0749-596X(03)00095-0

Unsworth, N., \& Engle, R. W. (2007). The nature of individual differences in working memory capacity: Active maintenance in primary memory and controlled search from secondary memory. Psychological Review, 114, 104-132. doi:10.1037/0033295X.114.1.104

Unsworth, N., Heitz, R. P., Schrock, J. C., \& Engle, R. W. (2005). An automated version of the operation span task. Behavior Research Methods, 37, 498-505. doi:10.3758/BF03192720 